\title{
UM ESTUDO BIBLIOMÉTRICO SOBRE O GRAFENO
}

\author{
Geanni Barbosa da Silveira e Silva Pessanha \\ Institutos Superiores de Ensino do Censa \\ Rua Salvador Correa, 139, Centro, Campos dos Goytacazes, RJ, Brasil. \\ gi_silveira@hotmail.com \\ Mara Regina dos Santos Barcelos \\ Institutos Superiores de Ensino do Censa \\ Rua Salvador Correa, 139, Centro, Campos dos Goytacazes, RJ, Brasil. \\ marabarceloss@gmail.com
}

\begin{abstract}
RESUMO
O grafeno tem ganhado grande visibilidade pela quantidade de propriedades que possui e pela possibilidade de aplicações nas mais diversas áreas, mesmo sendo um material descoberto a um certo tempo. Apesar do notório conhecimento de que o grafeno pode revolucionar a indústria, novos estudos são necessários, principalmente pela demanda em obtê-lo de formas tecnicamente e economicamente viáveis. Este trabalho tem o objetivo de realizar um levantamento bibliométrico, com o intuito de criar uma visão sistemática do tema em questão, identificando a escala de relevância dos seguintes quesitos: ano de publicação dos artigos; clusters de palavras-chave; idiomas; principais autores indicando o índice $\mathrm{H}$ e a relação entre eles; afiliação; países; áreas de conhecimento e periódicos. Para tanto foi realizada uma pesquisa na base de dados Scopus, no mês de maio do ano de 2019, por meio de pesquisa avançada tendo como estrutura: TITLE ("graphene"), desta maneira os registros foram selecionados quando os termos em questão faziam parte de seu título. O filtro (LIMIT TO (DOCTYPE, ar) foi usado a fim de se obter apenas artigos de periódicos. Os resultados revelaram que as publicações sobre o tema estão distribuídas por diversos autores, em vários países, indicando um crescimento constante da produção científica ao longo dos anos.
\end{abstract}

Palavra-chave: Nanomateriais; Grafeno; Propriedades. 


\begin{abstract}
Graphene has gained great visibility by the amount of properties it has and the possibility of applications in several areas, even being a material discovered at a certain time. Despite the notorious knowledge that graphene can revolutionize the industry, further studies are needed, mainly by the demand to obtain it in technically and economically viable ways. This paper aims to perform a bibliometric survey, in order to create a systematic view of the theme in question, identifying the relevance scale of the following issues: year of publication of the articles; keyword clusters; languages; main authors indicating the $\mathrm{H}$ index and the relationship between them; affiliation; countries; knowledge areas and journals. For this purpose, a survey was conducted in the Scopus database, in May of 2019, through advanced research with the structure: TITLE ("graphene"), in this way the records were selected when the terms in question were part of their title. The filter (LIMIT TO (DOCTYPE, ar) was used in order to obtain only journal articles. The results revealed that publications on the theme are distributed by several authors in many countries, indicating a constant growth of scientific production over the years.
\end{abstract}

Keywords: Nanomaterials; Graphene; Properties.

\title{
Como Citar:
}

SILVA, G.B.S. 1; BARCELOS, Mara Regina 2. Um Estudo Bibliométrico sobre o Grafeno. In: SIMPÓSIO DE PESQUISA OPERACIONAL E LOGÍSTICA DA MARINHA, 19., 2019, Rio de Janeiro, RJ. Anais [...]. Rio de Janeiro: Centro de Análises de Sistemas Navais, 2019. 


\section{INTRODUÇÃO}

O grafeno foi explorado pela primeira vez em 1947 pelo físico Philip Wallace, apenas como uma teoria, pois acreditava-se que uma estrutura bidimensional não poderia existir fisicamente. No entanto, o termo grafeno foi empregado pela primeira vez em 1987, sendo descrito como camadas planas monoatômicas soltas em uma estrutura grafítica e posteriormente consolidado pela International Union of Pure and Applied Chemistry (IUPAC) em 1994. Em 2004, um grupo do Centro de Nanotecnologia da Universidade de Manchester liderado pelos Prof. André K. Geim e Konstantin Novoselov, conseguiram produzi-lo através do isolamento de pequenos fragmentos das monocamadas, a partir do grafite (Graphene Science Handbook, 2016).

Nesse contexto, Araia (2012) apresenta o grafeno como um material revolucionário por ser extremamente forte, leve, flexível, ótimo condutor de eletricidade e quase totalmente transparente. Por apresentar propriedades peculiares e diversidade em suas formas estruturais, o grafeno possui forte tendência a diversas aplicações e têm desempenhado um papel significativo na ciência dos nanomateriais (ALIVISATOS, 2004). A partir da Figura 1 podese observar a relação das propriedades com as respectivas aplicabilidades.

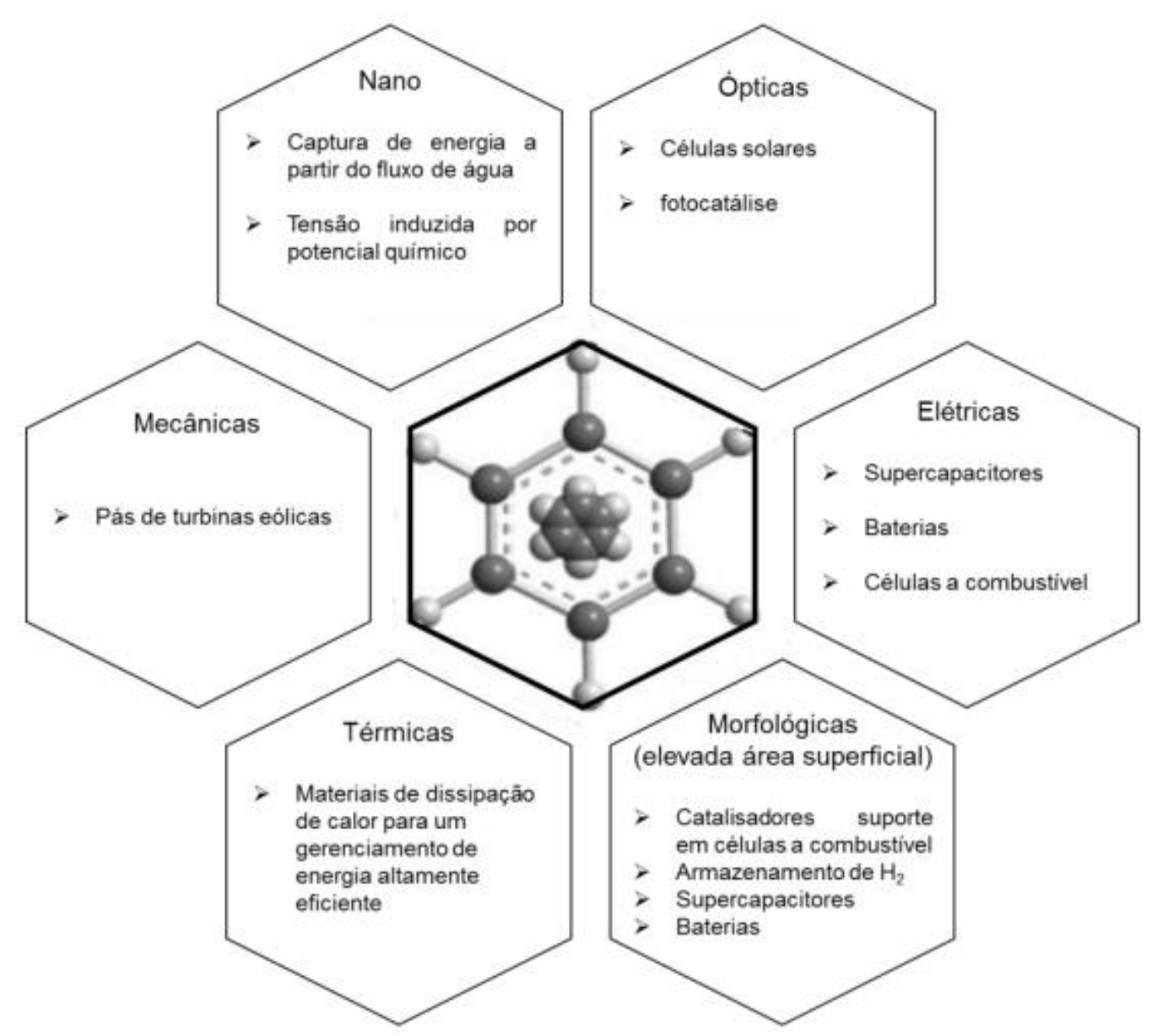

Figura 1: Relação entre as propriedades do grafeno e suas aplicações.

Fonte: Vieira Segundo; Vilar, 2017. 
Vieira Segundo e Vilar (2017) fizeram um comparativo em relação as propriedades pertencentes ao grafeno e outros materiais comumente utilizados, a saber, maior resistência mecânica que o aço, condutividade térmica mais elevada que o cobre, mobilidade eletrônica mais alta que o silício, maior área superficial que a observada para o grafite, além de uma leveza considerável. Por apresentar uma estrutura eletrônica distinta possui ainda a capacidade de reunir tais propriedades concomitantemente, podendo vir a substituir diversos materiais nas mais variadas aplicações. Tais aplicações incluem sensores, transistores, dispositivos eletrônicos portáteis e sistemas de armazenamento de energia eletroquímica, por exemplo.

No que tange a produção e desenvolvimento, há ainda a necessidade de aperfeiçoamento para melhoria contínua da qualidade, embora muitos métodos de síntese já tenham sido desenvolvidos. Além disso, a maioria dos métodos de caracterização recentes necessitam da transferência do grafeno a um substrato específico, o que diminui a eficiência da caracterização. Portanto, métodos de identificação rápida e eficazes ainda precisam ser desenvolvidos (NOVOSELOV et al., 2012).

Junior e Lobato (2017) através de uma revisão bibliográfica descreveram os principais métodos de obtenção do grafeno, aos quais pode-se destacar a esfoliação mecânica, esfoliação química e deposição química em fase de vapor.

No Brasil poucas são as iniciativas de pesquisa e desenvolvimento tecnológico, contudo há alguns exemplos nacionais de investimentos no intuito de melhorar a qualidade dos produtos e materiais, assim como a vida das pessoas. O MackGrafe, é um Centro de Pesquisas Avançadas em Grafeno, Nanomateriais e Nanotecnologia, que foi idealizado e obteve investimento da Universidade Presbiteriana Mackenzie. Suas atividades foram iniciadas em 2013 em São Paulo, com uma verba inicial de aproximadamente US\$ 15 milhões. O objetivo da pesquisa nesse centro é sintetização e caracterização do grafeno, bem como o desenvolvimento de dispositivos, principalmente relacionados à fotônica (MACKGRAFE, 2015).

Outra iniciativa foi adotada pela Universidade Federal de Minas Geral (UFMG), responsável por sediar um evento internacional sobre grafeno em 2010, onde reuniu especialistas no "International Graphene Conference - Graphene Brazil 2010". O mesmo foi promovido pela Universidade de Boston (EUA) e pelo Instituto Nacional de Ciência e Tecnologia (INCT) de Nanomateriais de Carbono e coordenado pela UFMG. O objetivo principal foi propiciar à comunidade científica brasileira a troca de informações sobre esse tema tão relevante (UFGM, 2010).

Ainda a UFMG e a Universidade de Rice (Texas) publicaram os resultados da otimização do método de esfoliação do grafite com a utilização de micro-ondas para a produção do grafeno, artigo ao qual resultou na capa da edição de maio da revista Journal of Brazilian Chemical Society (ARAÚJO, 2015). A UFMG tem cerca de 30 pesquisadores dedicados às pesquisas na área do grafeno e a universidade vem testando o método Chemical Vapor Deposition (CVD) para produzir o grafeno em escala industrial. Por meio desta técnica, é possível produzir um vapor de átomos de carbono que, quando depositados sobre um substrato de cobre, forma uma película de grafeno em cima do cobre (PIERRO, 2013). 


\section{METODOLOGIA}

A pesquisa bibliométrica foi realizada conforme indicado no modelo Webibliomining (COSTA, 2010), a fim de analisar a produção científica sobre o grafeno, considerando os seguintes tópicos: ano de publicação; tipos de documentos; clusters de palavras-chave; idiomas; principais autores; rede de autores e o índice $\mathrm{H}$; afiliação; países; áreas de conhecimento e periódicos.

A base de dados Scopus foi acessada por meio do Portal de Periódicos CAPES, no mês de maio do ano de 2019, ao qual optou-se pela pesquisa avançada. A estrutura utilizada foi TITLE ("graphene"). Desta maneira, os registros foram selecionados através da busca do termo (graphene) somente nos títulos dos mesmos, resultando em 103.622 registros, dos quais, 88.050 são artigos.

\section{RESULTADOS DA BIBLIOMETRIA}

Nesta seção são apresentados os resultados obtidos através da pesquisa bibliométrica para o termo "graphene" realizada na base de dados Scopus.

\section{1. ÍNDICES DE PUBLICAÇÕES POR ANO}

Os resultados mostram um crescimento constante em relação ao número de publicações sobre o tema em questão ao longo dos últimos dezesseis anos, conforme ilustra a Figura 2.

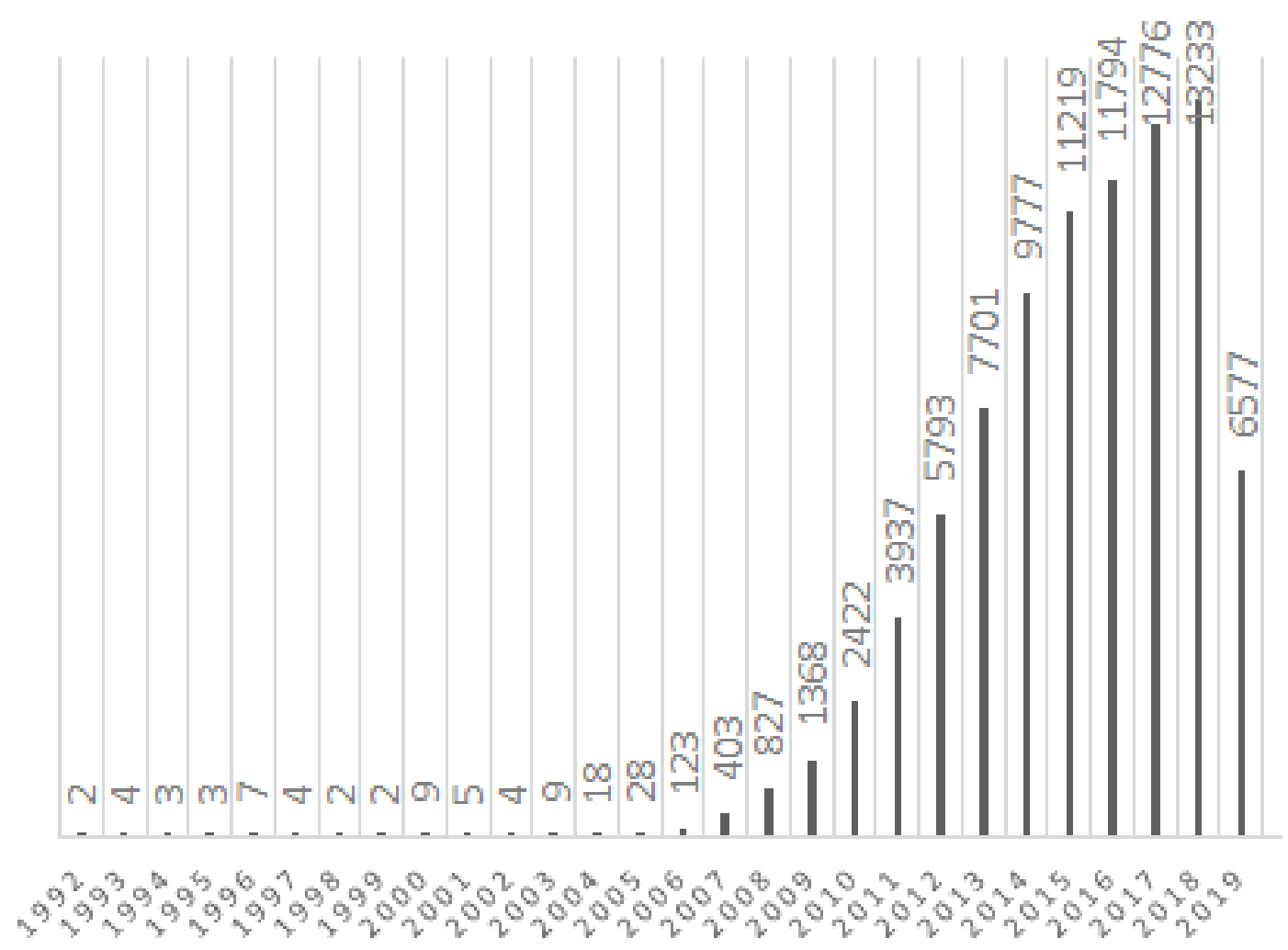

Figura 2: Publicações no período de 1992 a 2019.

Fonte: Elaborado pelos autores. 
A partir do gráfico gerado pode-se perceber que o ano com maior número de publicações é 2018, com 13.233 registros, visto que referente ao ano de 2019 o número de publicações é parcial, pela data de realização da pesquisa. Outro aspecto interessante, é que desde que as publicações sobre o tema iniciaram (1992), não houveram períodos sem publicações.

\subsection{TIPOS DE DOCUMENTOS E IDIOMAS}

Em relação aos tipos de documentos, foram encontrados 103.622 registros inicialmente, dos quais, 88.050 artigos em periódicos, 10.394 artigos de congressos, 2.282 revisões e 1.033 capítulos de livros. Os outros registros possuem menos de 1.000 publicações e não foram citados.

No quesito idiomas, considerando somente os artigos de periódicos, o inglês predomina com 85.966 registros $(97,63 \%)$, seguido por 1.877 registros em chinês. O restante dos idiomas possui menos de 100 registros publicados.

\subsection{CLUSTER DE PALAVRAS-CHAVE}

Para gerar o cluster de palavras-chave no software VOSviwer foi necessário utilizar um arquivo exportado pela própria base de dados, a base Scopus. O limite para exportação dessa base é de 2.000 de registros. Neste contexto, devido à grande quantidade de registros alcançados pela busca, foram selecionados os artigos publicados nos últimos 5 anos, com no mínimo 200 citações (o mesmo procedimento foi utilizado para gerar a rede de autores).

Foram analisadas 1.596 palavras-chaves, das quais, 108 atenderam ao limite mínimo de 5 ocorrências nos artigos em questão. A Figura 3 ilustra a intensidade de ocorrências dessas palavras-chaves e a palavra-chave com maior índice é graphene, totalizando 124 ocorrências.

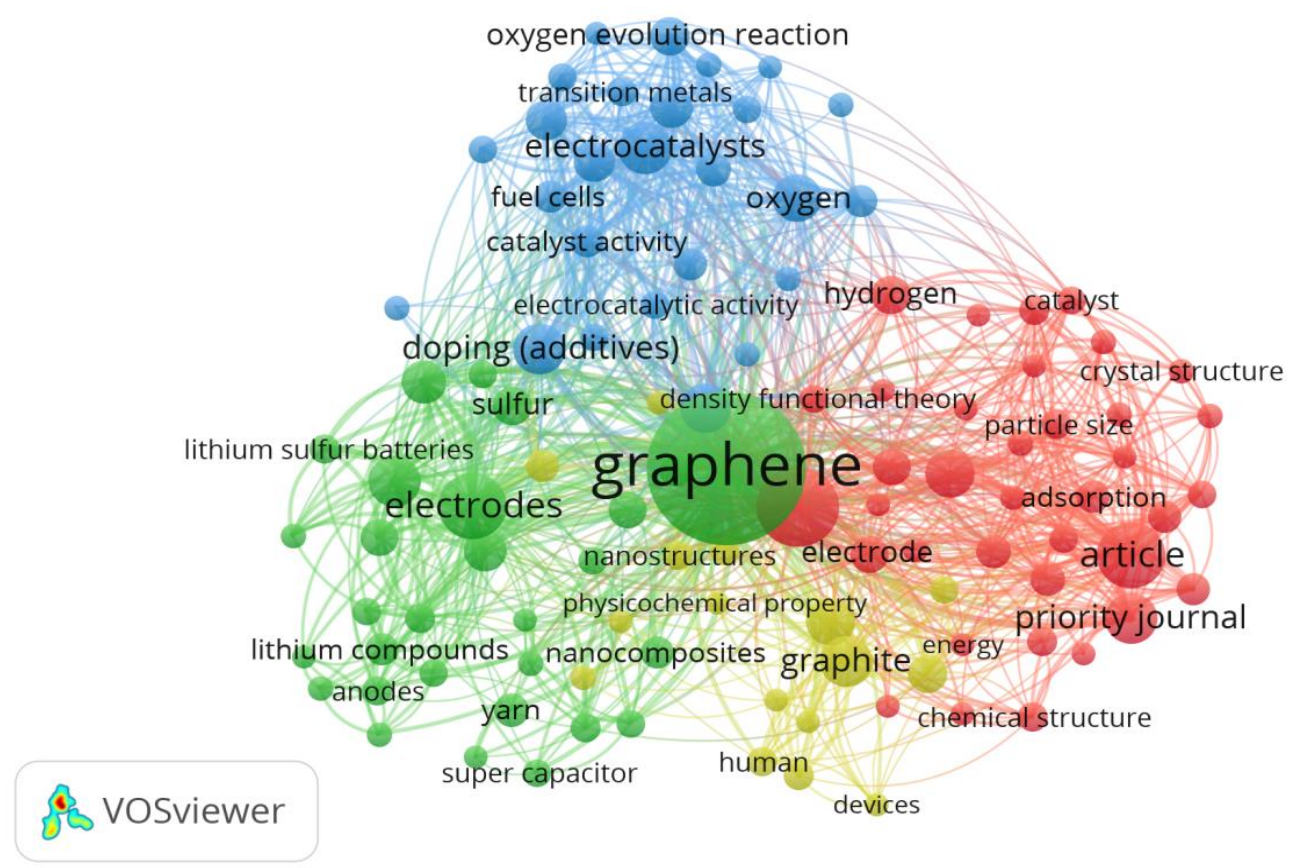

Figura 3: Cluster de palavras-chave.

Fonte: VOSviwer. 


\subsection{IÍNDICES DE PUBLICAÇÕES POR PAÍSES}

O número de publicações por países pode ser observado na Tabela 1. A China aparece em primeiro lugar, totalizando 38.892 registros (aproximadamente $44 \%$ do total de artigos), enquanto o Brasil aparece na décima oitava posição com 1.169 registros publicados. É importante ressaltar que foram ilustrados na tabela apenas os países com pelo menos 1.000 artigos publicados.

Tabela 1: Índice de publicações por países

\begin{tabular}{l|c}
\hline \multicolumn{1}{c}{ Países } & $\mathbf{N}^{\mathbf{0}}$ de publicações \\
\hline China & 38.892 \\
\hline Estados Unidos & 13.727 \\
\hline Coreia do Sul & 7.457 \\
\hline Índia & 5.745 \\
\hline Irã & 4.408 \\
\hline Japão & 3.692 \\
\hline Alemanha & 3.570 \\
\hline Reino Unido & 3.272 \\
\hline Cingapura & 2.373 \\
\hline Espanha & 2.362 \\
\hline Austrália & 2.196 \\
\hline Taiwan & 2.138 \\
\hline Itália & 2.128 \\
\hline Federação Russa & 2.101 \\
\hline França & 1.983 \\
\hline Canada & 1.542 \\
\hline Hong Kong & 1.270 \\
\hline Brasil Elaborado pelos autores. \\
\hline Malásia & 1.169 \\
\hline
\end{tabular}

\section{5. ÍNDICES DE PUBLICAÇÕES POR ÁREAS DE CONHECIMENTO}

A Tabela 2 relaciona o número de publicações por área de conhecimento, com destaque para Ciências de Materiais, com 49.067 registros, seguido pela área de Química com 38.880 registros. Vale ressaltar que foram ilustradas apenas áreas de conhecimento com no mínimo 2.000 registros.

Tabela 2: Índice de publicações por área de conhecimento

\begin{tabular}{l|c}
\multicolumn{1}{c}{ Área de conhecimento } & $\mathbf{N}^{\mathbf{0}}$ de publicações \\
\hline Ciência de materiais & 49.067 \\
\hline Química & 38.880 \\
\hline Física e Astronomia & 35.665 \\
\hline Engenharia & 23.621 \\
\hline Engenheiro químico & 18.873 \\
\hline Energia & 6.741 \\
\hline Bioquímica, Genética e Biologia Molecular & 5.500 \\
\hline Ciência ambiental & 3.630 \\
\hline
\end{tabular}

Fonte: Elaborado pelos autores. 


\section{6. ÍNDICES DE PUBLICAÇÕES POR PERIÓDICOS}

A Tabela 3 indica o número de publicações por periódico, com destaque para o Rsc Advances com 3.355 registros. Os periódicos com menos de 2.000 publicações não foram citados na tabela.

Tabela 3: Índice de publicações por periódicos

\begin{tabular}{l|c}
\hline \multicolumn{1}{c|}{ Periódicos } & $\mathbf{N}^{\mathbf{0}}$ de publicações \\
\hline Rsc Advances & 3.355 \\
\hline Physical Review B Condensed Matter And Materials Physics & 3.133 \\
\hline Carbon & 2.897 \\
\hline Applied Physics Letters & 2.336 \\
\hline ACS Applied Materials And Interfaces & 2.014 \\
\hline \multicolumn{2}{c}{ Fonte: Elaborado pelos autores. }
\end{tabular}

\section{7. ÍNDICES DE PUBLICAÇÃO POR AUTORES}

A Tabela 4 ilustra a relação entre o número de publicações por autores, com destaque para os autores: Taniguchi, T. com 334 publicações e Watanabe, K. com 329. Além disso, é informado o índice-H dos autores com destaque para os autores: Ruoff, R.S. (137) e Tour, J.M. (124).

Tabela 4: Índice de publicações por autores, incluindo o índice $\mathrm{H}$

\begin{tabular}{c|c|c}
\hline Autores & $\mathbf{N}^{\mathbf{0}}$ de publicações & Índice H \\
\hline Taniguchi, T. & 334 & 81 \\
\hline Watanabe, K. & 329 & 83 \\
\hline Peeters, F.M. & 228 & 83 \\
\hline Ruoff, R.S. & 207 & 137 \\
\hline Novoselov, K.S. & 182 & 112 \\
\hline Pumera, M. & 180 & 77 \\
\hline Feng, X. & 174 & 93 \\
\hline Tour, J.M. & 170 & 124 \\
\hline Chen, S.M. & 168 & 58 \\
\hline Katsnelson, M.I. & 161 & 91 \\
\hline Liu, Z. & 160 & 87 \\
\hline Zhu, H. & 156 & 66 \\
\hline
\end{tabular}

Fonte: Elaborado pelos autores.

Como complemento, a Figura 4 apresenta a rede de autores com sete clusters. O maior cluster possui doze autores conectados. Foram identificados 820 autores, dos quais, 8 atenderam ao limite mínimo de 5 ocorrências nos artigos em questão. 


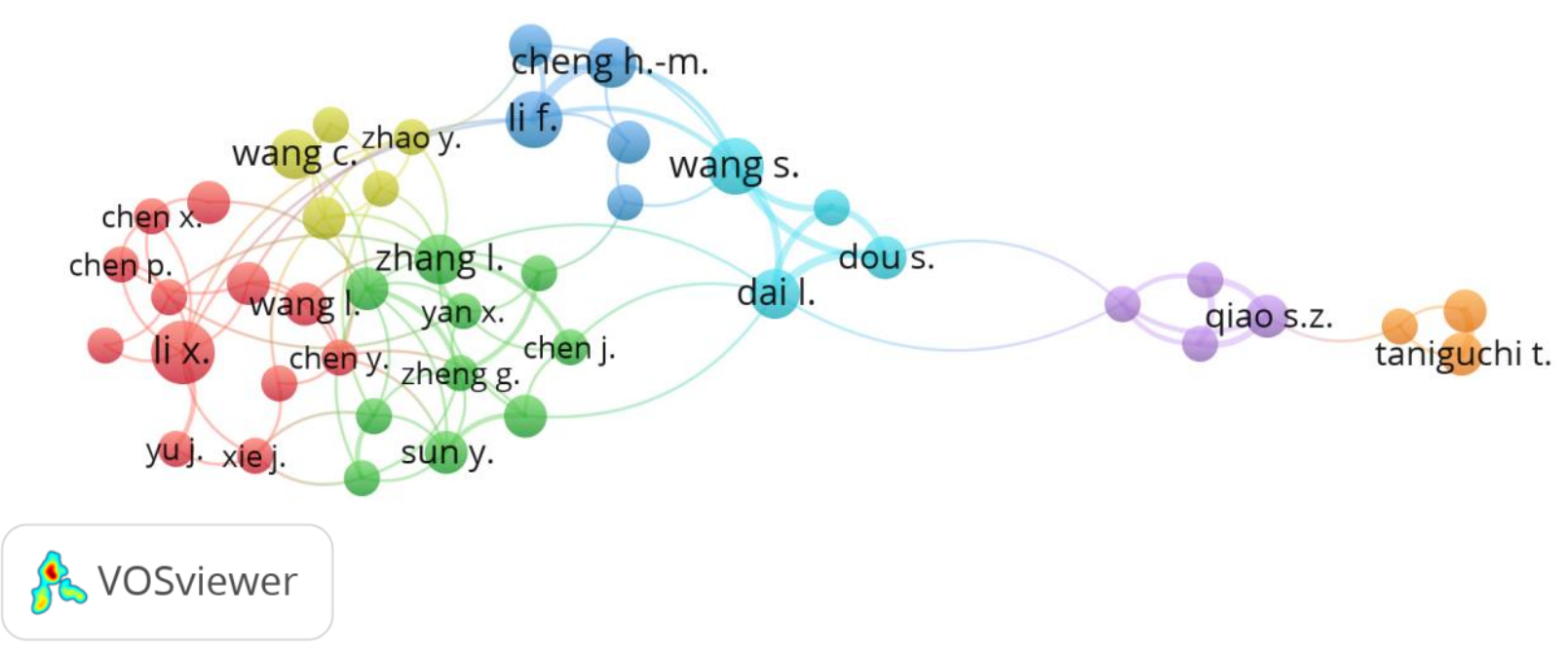

Figura 4: Rede de autores.

Fonte: VOSviwer.

\subsection{IINDICE DE PUBLICAÇÕES POR AFILIAÇÃO}

A Tabela 5 indica o número de publicações por afiliação, com destaque para a Chinese Academy of Sciences (5.998) e Ministry of Education China (5.645). As instituições citadas na tabela possuem no mínimo 1.000 artigos publicados.

Tabela 5: Índice de publicação por afiliação

\begin{tabular}{l|c}
\hline \multicolumn{1}{c}{ Instituições } & $\mathbf{N}^{\mathbf{0}}$ de publicações \\
\hline Chinese Academy of Sciences & 5.998 \\
\hline Ministry of Education China & 5.645 \\
\hline Tsinghua University & 1.536 \\
\hline University of Chinese Academy of Sciences & 1.371 \\
\hline Nanyang Technological University & 1.272 \\
\hline University of Science and Technology of China & 1.045 \\
\hline Peking University & 1.039 \\
\hline Zhejiang University & 1.032 \\
\hline
\end{tabular}

Fonte: Elaborado pelos autores.

\section{CONCLUSÕES}

A pesquisa na base de dados Scopus revelou que as publicações sobre o tema estão distribuídas por diversos autores, em vários países, indicando um crescimento constante da produção científica ao longo dos anos.

$\mathrm{O}$ autor Taniguchi, T. aparece em destaque, com 334 publicações (índice- $\mathrm{H}=81$ ), sendo que o maior índice-H é 137 do autor Ruoff, R.S., que possui 207 publicações. A rede de autores mostra a formação de 7 núcleos entre os autores, sendo que o maior deles possui 12 autores.

Em relação ao índice de publicação por países, se destacam China e EUA. A Língua Inglesa predomina em relação a outros idiomas, e a China é o país que apresenta maior 
quantidade de publicações. E ainda, o tipo de documento com maior quantidade de registros é artigos de periódicos.

Em relação à área de pesquisa, Ciências de materiais aparece em primeiro lugar, com maior número de publicações (49.067). Quanto a afiliação, a Chinese Academy of Sciences se destaca com 5.998 autores. Já o periódico com maior número de publicações é Rsc Advances com 3.355 registros.

Como limitação do trabalho pode-se citar que não é possível generalizar os resultados, visto que a bibliometria considerou apenas a base de dado Scopus. Neste sentido, pesquisas em outras bases podem realizadas como trabalhos futuros, a fim de observar a evolução das publicações sobre o grafeno.

\section{REFERÊNCIAS BIBLIOGRÁFICAS}

[1] ARAIA, Eduardo. Como Grafeno vai mudar sua vida. Revista Planeta, 472.ed., jan. 2012.

[2] ARAÚJO, Ana Rita. Na corrida pelo grafeno. 2015. Disponível em: https://www.ufmg.br/boletim/bol1904/5.shtml. Acesso em: 9 out. 2019.

[3] ALIVISATOS, A.P. Menos é Mais na Medicina. Scientific American Brasil, São Paulo, n. 22, p. 74-81, Edição Especial, mar. 2004.

[4] COSTA, H.G. (2010), "Model for webibliomining: proposal and application", Revista da FAE, Vol. 13 No. 1, pp. 115-126.

[5] JUNIOR, M. A. P.; LOATO, A. K. C. O grafeno: meios de obtenção e possíveis aplicações na indústria automotiva. 2017.

[6] ROCHA, J.P.M. Grafeno como fonte de energia renovável: um estudo prospectivo. 2015.

[7] MACKGRAFE Mackenzie. Sobre. Disponível em: http://mackgrafe.mackenzie.br/mackgrafe_about.html?\&L=1 Acesso em: 7 maio 2019.

[8] MAHMOOD Aliofkhazraei, Nasar Ali, William I. Milne, Cengiz S. Ozkan, Stanislaw Mitura \& Juana L. Gervasoni. Graphene Science Handbook, Six-Vol. 2016, (em inglês) ISBN 9781466591196.

[9] NOVOSELOV, K. S. et al. A roadmap for graphene. Nature, v.190, out. 2012. p.192200.

[10] PIERRO, B. O grafeno e seus desafios. 2013. Disponível em: http://revistapesquisa.fapesp.br/2013/12/18/o-grafeno-e-seus-desafios/. Acesso em: 9maio 2019.

[11] UFMG. Evento internacional sobre grafeno reúne especialistas em BH. 2010. Disponível em: https://www.ufmg.br/online/arquivos/017642.shtml. Acesso em: 8 maio. 2019.

[12] VIEIRA SEGUNDO, J. E. D.; Vilar, E. O. Grafeno: Uma revisão sobre propriedades, mecanismos de produção e potenciais aplicações em sistemas energéticos. Revista Eletrônica de Materiais e Processos, v. 11, n. 2 (2016) 54-57 ISSN 1809-879, 2017. 